\title{
Post-operative chemotherapy improves the survival of patients with well differentiated widespread metastatic appendiceal non-mucinous adenocarcinomas
}

\author{
Tianyu Yu ${ }^{1 \#}$, Hao Fan ${ }^{1 \#}$, Qiang $\mathrm{Lv}^{1}$, Jun Li ${ }^{1}$, Lei Wei ${ }^{1}$, Minglong Wang ${ }^{2}$, Jiangnan Dong ${ }^{1}$, Xiaoyan Cai $^{1 \wedge}$ \\ ${ }^{1}$ Department of General Surgery, Pudong New Area Gongli Hospital Affiliated to Naval Military Medical University, Naval Military Medical \\ University, Shanghai, China; ${ }^{2}$ Department of Plastic Surgery, Pudong New Area Gongli Hospital Affiliated to Naval Military Medical University, \\ Naval Military Medical University, Shanghai, China \\ Contributions: (I) Conception and design: X Cai, T Yu, J Dong; (II) Administrative support: None; (III) Provision of study materials or patients: None; \\ (IV) Collection and assembly of data: H Fan, L Wei, M Wang; (V) Data analysis and interpretation: T Yu, H Fan, J Li; (VI) Manuscript writing: All \\ authors; (VII) Final approval of manuscript: All authors. \\ "These authors contributed equally to this work. \\ Correspondence to: Xiaoyan Cai. Department of General Surgery, Pudong New Area Gongli Hospital Affiliated to Naval Military Medical University, \\ Naval Military Medical University, Shanghai 200135, China. Email: cxy6429@163.com.
}

Background Appendiceal adenocarcinoma is a very rare type of tumor, often asymptomatic in the early
stages of development. Surgical resection is the most preferred intervention against appendiceal non-
mucinous adenocarcinoma, but the efficacy of post-operative adjuvant chemotherapy is still unclear because
the cancer is rare. Accordingly, we sought to characterize appendiceal non-mucinous adenocarcinoma profile
that confers a better survival advantage for post-operative chemotherapy.
Methods: We analyzed patients with appendiceal non-mucinous adenocarcinoma in the Surveillance,
Epidemiology and End Results database, histologically diagnosed for the cancer between 2004 and 2015.
Nearly half of the patients first underwent surgery and thereafter received post-operative chemotherapy.
Logistic regression, Kaplan-Meier, univariate and multivariate Cox analysis were performed to evaluate the
odds ratio for the propensity of patients underweening chemotherapy, whereas hazard ratios were used to
evaluate the overall as well as cancer-specific survival. Results: Of the 724 patients with appendiceal non-mucinous adenocarcinoma who underwent surgery, $301(41.6 \%)$ received post-operative chemotherapy. Notably, patients with metastatic appendiceal nonmucinous adenocarcinoma were more likely to receive chemotherapy (OR =7.42, 95\% CI: 5.34-10.39, $\mathrm{P}<0.001$ ), similar to those with poor pathologically differentiated cancer types (OR =2.10, 95\% CI: 1.49-3.00, $\mathrm{P}<0.001)$. However, univariate and multivariable Cox regression analyses found no significant overall survival and cancer-specific survival advantage for patients put on postoperative post-operative chemotherapy. In the disease stage and pathological differentiation groups, only patients with widespread metastatic (Stage IV) but well differentiated tumors displayed better 3-year (11.9\% in overall survival, $11.5 \%$ in cancer-specific survival) and 5 -year survival rate (7.8\% in overall survival, $6.8 \%$ in cancer-specific survival) to post-operative chemotherapy.

Conclusions: Staging and grading of appendiceal non-mucinous adenocarcinoma is invaluable in guiding the rationale of post-operative chemotherapy. Findings of this research support the view that only patients with both of widespread metastatic but well differentiated appendiceal non-mucinous adenocarcinoma should be considered for post-operative chemotherapy. Nonetheless, further prospective multidisciplinary clinical trials are necessary to further discern the use chemotherapy after surgery in appendiceal non-mucinous adenocarcinoma patients.

^ ORCID: Tianyu Yu 0000-0001-7353-0262; Xiaoyan Cai 0000-0001-9926-904X. 
Keywords: Appendiceal non-mucinous adenocarcinoma; SEER; chemotherapy; overall survival; cancer specific survival

Submitted Nov 12, 2020. Accepted for publication Mar 19, 2021.

doi: 10.21037/apm-20-2233

View this article at: http://dx.doi.org/10.21037/apm-20-2233

\section{Introduction}

Appendiceal malignancies are rare tumors, only pathologically identified in about $0.9 \%$ to $1.4 \%$ of surgical appendectomy specimens (1-3). In addition, more than $30 \%$ of appendiceal cancer patients present with local or distant metastases at the time of diagnosis (1). Appendiceal malignant tumors are generally divided into non-epithelial and epithelial type. Non-epithelial neoplasms include carcinoids, lymphomas, sarcoma and adenocarcinomas. Adenocarcinomas, further divided in to mucinous and non-mucinous types, account for the majority of epithelial tumors. Overall, adenocarcinomas rank second among all tumors, only behind carcinoid (4). Meanwhile, appendiceal mucinous adenocarcinoma (AMA) has been implicated for pseudomyxoma peritonei (PMP), characterized by progressive accumulation of mucous secreting tumor cells in the abdomen and pelvis (5-7). Meanwhile, the local invasive and metastatic blueprints of appendiceal non-mucinous adenocarcinomas (ANMA) are comparable to those of colorectal adenocarcinomas (8).

Currently, surgical resection remains the first-choice treatment against appendiceal malignant tumor, with appendectomy or colectomy recommended for patients with distinct diameter appendiceal carcinoid tumors (9). On the other hand, cytoreductive surgery (CRS) is preferred against AMA (10). The effect of systemic chemotherapy in multiple appendiceal adenocarcinomas has been extensively studied. In one study, based on the National Cancer Database (NCDB) encompassing 639 patients, Lu et al. reported against systemic chemotherapy in patients with stage IV and low-grade AMA (11). Elsewhere, Lieu et al. found systemic chemotherapy improved the prognosis of appendiceal signet-ring adenocarcinoma (12). Further to this, one study based on a large population dataset showed that systemic chemotherapy improves overall survival (OS) of patients with poorly differentiated AMA (13). In a separate but related study, Uemura et al. observed that systemic chemotherapy improved the survival of patients with stage IV ANMA (14).

In recent years, combination interventions have been embraced against appendiceal malignant tumor. Indeed, studies show that a combination of CRS and adjuvant therapy significantly improves the 5 -year survival rate of AMA patients by $20 \%$ to $86 \%(15,16)$. However, because ANMA is a rare cancer, the prognostic value of postoperative chemotherapy against the disease is unclear. Because of the pathological similarity between ANMA and colorectal adenocarcinomas, the rationale for ANMA chemotherapies mimics that of colorectal cancer (CRC) (17). However, the efficacy of this rationale has never been validated because of limited ANMA cases. Even so, superimposing CRC interventions to CRC may be counterproductive due to the distinct molecular signatures between the two cancer types $(18,19)$. Thus, the specific category of ANMA patients may benefit from postoperative chemotherapy remains unclear.

In this study, we used The Surveillance, Epidemiology and End Results (SEER) data to evaluate the prognosis differences of post-operative chemotherapy among ANMA patients. Our findings will guide the rationale of postoperative use of chemotherapy in ANMA. We present the following article in accordance with the STROBE reporting checklist (available at http://dx.doi.org/10.21037/apm-202233).

\section{Methods}

\section{Inclusion and exclusion criteria}

The SEER database is currently the largest publicly available cancer consortium, with $26 \%$ of the data generated from American population (20-23). Relevant data was retrieved using SEER*Stat 8.3.6.1 program: SEER 18 Regs customs data (with additional treatment field), performed on Nov 2018 Sub (1973-2016 varying). Patients included in this study were diagnosed with either ANMA (ICD-O-3 code: $8140 / 3, \mathrm{n}=860$ ) or AMA (ICD-O-3 code: 8480/3, $\mathrm{n}=1,748$ ) between 2004 and 2015, with a view of comparing the survival difference between the two groups. We also aimed at evaluating the prognostic efficacy of post-operative chemotherapy among different ANMA sub-groups, albeit 
A

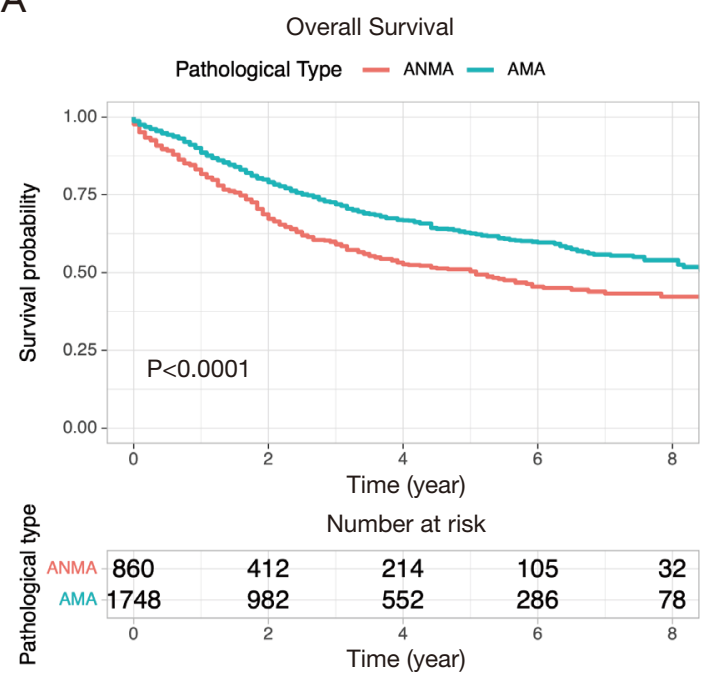

B

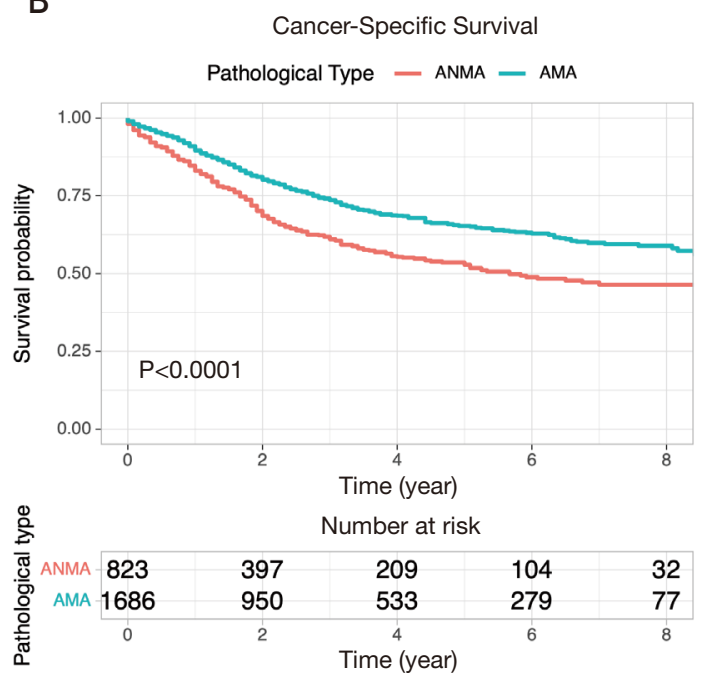

Figure 1 The comparative survival rates of patients with appendiceal adenocarcinoma subtypes (AMA versus ANMA). (A) Overall and (B) cancer-specific. AMA, appendiceal mucinous adenocarcinoma; ANMA, appendiceal non-mucinous adenocarcinomas.

aged between 18 and 90 years. Patients with multiple primary tumors, unclear diagnostic method, missing survival information, unknown TNM stage or grade, and already receiving radiotherapy were all excluded from the study. In the end, 721 ANMA patients satisfying the inclusion criteria were included in the final analyses.

Biodata such as age, race, sex, marital status together with additional information such as insurance status, pathological grade, TNM stage, types of surgery and chemotherapy were all captured. Patients were divided into either "chemotherapy" or "non-chemotherapy" groups based on whether or not they received chemotherapy after surgery. Regarding age at diagnosis, patients were stratified into five categories: younger than 50 years, between $50-59$ years, 60-69 years, 70-79 years and older than 80 years. On Insurance, patients were divided into groups of "Medicaid", "Insured", "Uninsured" and "Unknown". For marital status, "Divorced" and "Widowed" were grouped as "Single". Patients were also grouped according to race, which encompassed whites, blacks and other groups according to their race. Surgical types were classified into "Appendectomy alone", "Partial colectomy" and "Unconventional surgery". TNM staging was performed based on the $6^{\text {th }}$ American Joint Committee on Cancer (AJCC) guidelines, which groups TNM into non-metastatic (Stage I-II) and metastatic (Stage III-IV). Pathological grades consisted of well differentiated and poorly differentiated tumors. The primary endpoints in this study were OS and cancer-specific survival (CSS), with deaths attributed to ANMA the only parameter included in CSS analysis.

\section{Statistical analysis}

Logistic regression analysis was performed to determine the propensity of patients to undergo chemotherapy. OS and CSS of patients with different stages and grades of ANMA were analyzed using log-rank tests after Kaplan-Meier analyses. On the other hand, prognostic factors for the cancer were analyzed using univariate and multivariate Cox analyses. $\mathrm{P}$ value $\leq 0.05$ was considered to be statistically significant. All statistical analyses were performed using $\mathrm{R}$ software, version 4.0.2 (https://www.r-project.org). The study was conducted in accordance with the Declaration of Helsinki (as revised in 2013).

\section{Results}

\section{Demographic and clinical characteristics of ANMA patients}

Comparative Survival analysis between ANMA and AMA patients showed that the OS and CSS of ANMA patients was significantly lower than that of AMA patients (Figure 1). Of the 724 ANMA patients who underwent surgery, 301 of them were put on chemotherapy. Demographic and clinical characteristics of these patients are summarized in Table 1. Generally, the likelihood of receiving chemotherapy 
Table 1 Characteristics of patients and the likelihood of receiving post-operative chemotherapy, stratified by demographic factors

\begin{tabular}{|c|c|c|c|c|c|}
\hline Categories & \multicolumn{3}{|c|}{ Adjuvant chemotherapy } & OR $(95 \% \mathrm{Cl})$ & $P$ value \\
\hline \multicolumn{6}{|l|}{ Age (years) } \\
\hline$<50$ & $146(20.2)$ & $66(15.6)$ & $80(26.6)$ & Reference & Reference \\
\hline $50-59$ & $170(23.5)$ & $84(19.9)$ & $82(27.2)$ & $0.81(0.51-1.26)$ & 0.341 \\
\hline $70-79$ & $125(17.3)$ & $80(18.9)$ & $45(15.0)$ & $0.46(0.28-0.75)$ & 0.002 \\
\hline$\geq 80$ & $82(11.3)$ & $76(18.0)$ & $6(2.0)$ & $0.07(0.02-0.14)$ & $<0.001$ \\
\hline \multicolumn{6}{|l|}{ Insurance } \\
\hline Any medicaid & $47(6.5)$ & $33(7.8)$ & $14(4.7)$ & Reference & Reference \\
\hline Unknown & $140(19.3)$ & $71(16.8)$ & $69(22.9)$ & $2.29(1.15-4.77)$ & 0.022 \\
\hline \multicolumn{6}{|l|}{ Sex } \\
\hline Female & $356(49.2)$ & $202(47.8)$ & $154(51.2)$ & Reference & Reference \\
\hline Male & $368(50.8)$ & $221(52.2)$ & $147(48.8)$ & $0.87(0.65-1.17)$ & 0.366 \\
\hline \multicolumn{6}{|l|}{ Race } \\
\hline Black & $91(12.6)$ & $50(11.8)$ & $41(13.6)$ & Reference & Reference \\
\hline White & $587(81.1)$ & $347(82.0)$ & $240(79.7)$ & $0.84(0.54-1.32)$ & 0.453 \\
\hline Other & $46(6.4)$ & $26(6.1)$ & $20(6.6)$ & $0.94(0.46-1.91)$ & 0.861 \\
\hline Hemicolectomy & 434 (59.9) & $243(57.4)$ & $191(63.5)$ & Reference & Reference \\
\hline Others & $68(9.4)$ & $38(9.0)$ & $30(10.0)$ & $1.00(0.60-1.68)$ & 0.987 \\
\hline Appendectomy alone & $222(30.7)$ & $142(33.6)$ & $80(26.6)$ & $0.72(0.51-1.00)$ & 0.05 \\
\hline \multicolumn{6}{|l|}{ Disease stages } \\
\hline I-II (non-metastasis) & $418(57.7)$ & $325(76.8)$ & $93(30.9)$ & Reference & Reference \\
\hline III-IV (metastasis) & 306 (42.3) & $98(23.2)$ & $208(69.1)$ & 7.42 (5.34-10.39) & $<0.001$ \\
\hline \multicolumn{6}{|l|}{ Pathological grade } \\
\hline Well differentiation & $557(76.9)$ & 349 (82.5) & $208(69.1)$ & Reference & Reference \\
\hline Poor differentiation & $167(23.1)$ & $74(17.5)$ & $93(30.9)$ & $2.10(1.49-3.00)$ & $<0.001$ \\
\hline
\end{tabular}


A

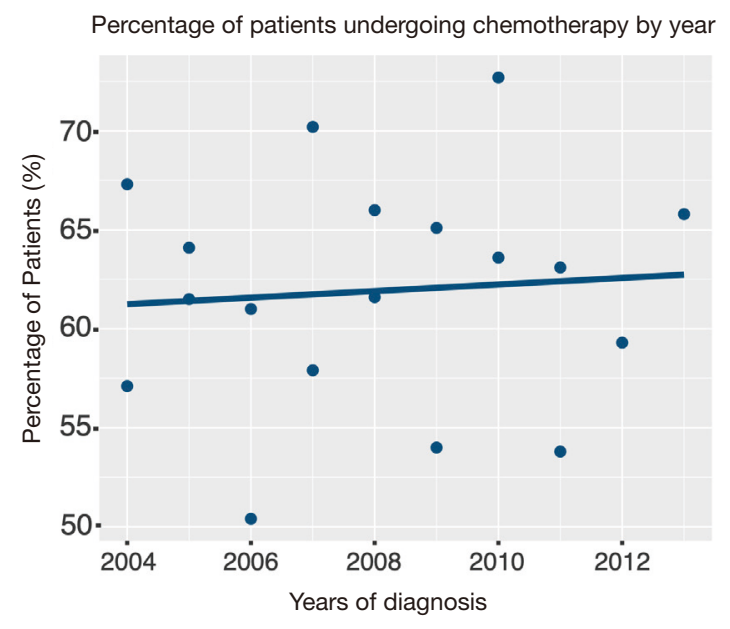

B

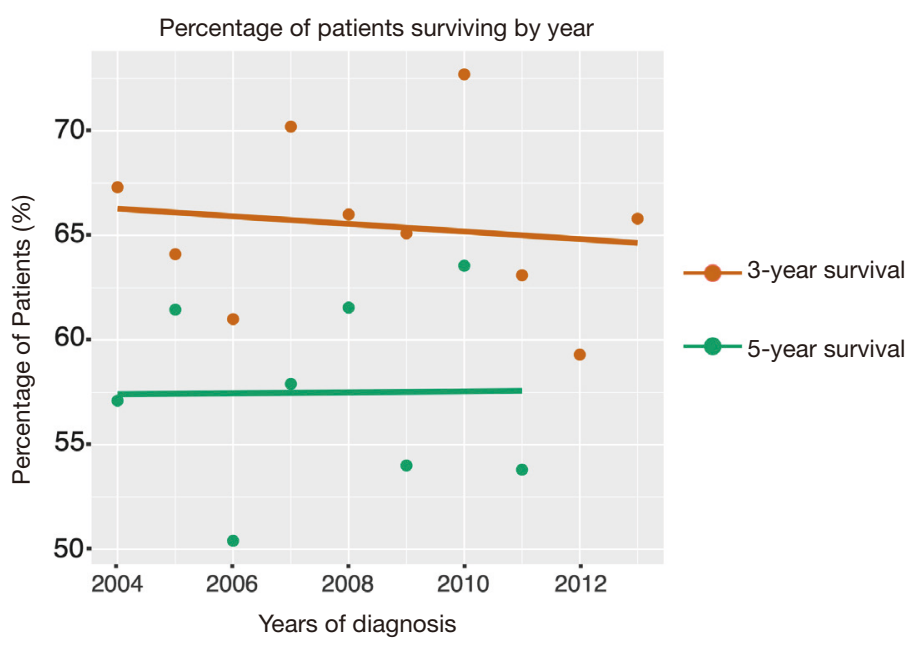

Figure 2 The trend in patients undergoing post-operative chemotherapy. (A) The 3-year and (B) 5-year survival rates.

decreases with age. At the same time, patients with metastasis cancers were 7.42 times more likely to receive chemotherapy, converse to those with non-metastatic ANMA (95\% CI: 5.34-10.39, $\mathrm{P}<0.001)$. In addition, patients with worse pathological differentiation $(\mathrm{OR}=2.10,95 \% \mathrm{CI}$ : 1.49-3.00, $\mathrm{P}<0.001)$, who underwent partial colectomy as opposed to (hemicolectomy) ( $\mathrm{OR}=0.72,95 \%$ CI: $0.51-1.00$, $\mathrm{P}=0.05)$, married and those with unknown insurance status were all more likely to receive chemotherapy.

\section{Trend in post-operative chemotherapy use and related survival of ANMA patients}

Between 2004 and 2015, the proportion of patients who received post-operative chemotherapy increased steadily (Figure $2 A$ ). However, over the same period, the 3 -year survival rate of ANMA patients decreased slightly, but the 5 -year survival rate remained relatively stable (Figure $2 B$ ).

\section{Prognostic factors for ANMA}

Univariate and multivariate Cox regression analyses revealed that age at diagnosis, marital status, TNM stage, type of surgery and pathologic grade were all independent factors for OS and CSS of ANMA patients (Table 2). Further multivariate cox regression analyses showed older age, higher disease stage, worse histological grade and receiving unconventional surgery were all associated with poor OS and CSS. In addition, the survival curve revealed that patients receiving chemotherapy were more likely to die than their counterparts (HR $=1.60$ in OS, 95\% CI: 1.28-2.00, $\mathrm{P}<0.001$; HR $=1.93$ in CSS, 95\% CI: $1.51-2.48$, $\mathrm{P}<0.001$ ) (Figure 3), suggesting that patients undergoing chemotherapy most likely present with advanced stages of ANMA. However, multivariate Cox analysis found no significant difference in OS and CSS between patients who received or did not receive chemotherapy.

\section{Groups of ANMA patients that benefit from post-operative chemotherapy}

Because the disease stage was the most significant determining factor for the likelihood to receive chemotherapy, we further analyzed the effect of chemotherapy in metastatic and non-metastatic ANMA. We found that post-operative chemotherapy offered benefit to ANMA patients with metastatic ANMA (Figure 4), in which the 3 -year OS rate increased from $37.9 \%$ to $45.2 \%$, whereas CSS increased from $38.5 \%$ to $45.3 \%$. However, there was little improvement on the 5 -year survival rate ( $32.5 \%$ to $33.6 \%$ for OS and $32.2 \%$ to $34 \%$ for CSS). Similar to metastatic status, pathologic grade was another key determining factor for chemotherapy. As such, we further analyzed the effectively of chemotherapy under the two pathological groups. Poorly differentiated, metastatic ANMA significantly decreased the OS and CSS by more than two folds, compared with well differentiated, nonmetastatic tumors (Figure 5). For non-metastatic types, 
Table 2 Univariable and Multivariate analysis for the overall and cancer-specific survival of ANMA patients stratified along age, insurance, sex, race, marital status, disease stage, surgical type, pathologic grade and post-operative chemotherapy

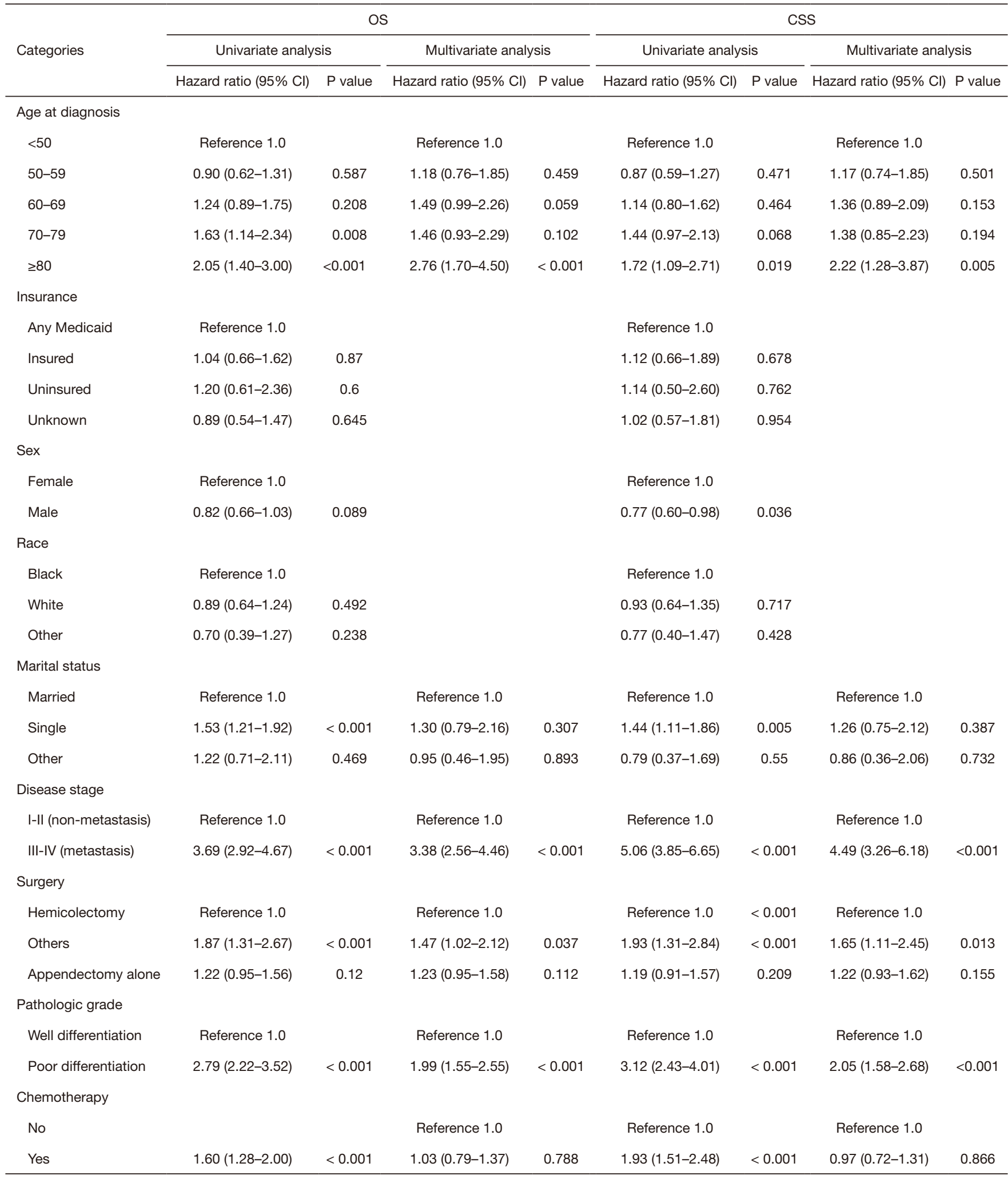

ANMA, appendiceal non-mucinous adenocarcinomas; OS, overall survival; CSS, cancer-specific survival. 
A
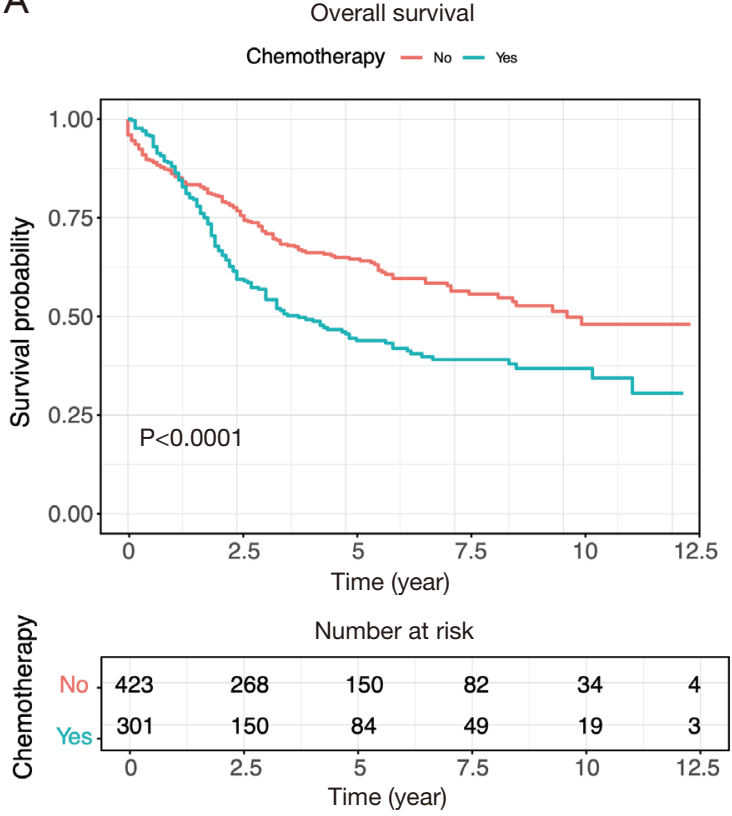

B

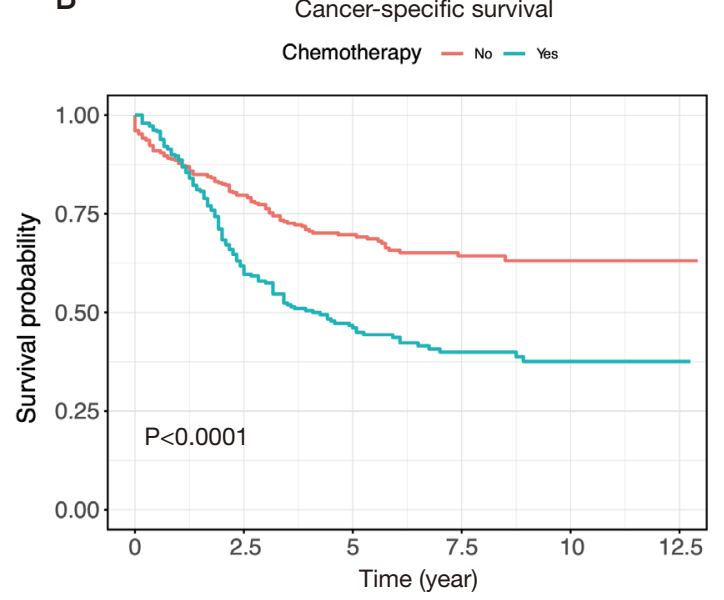

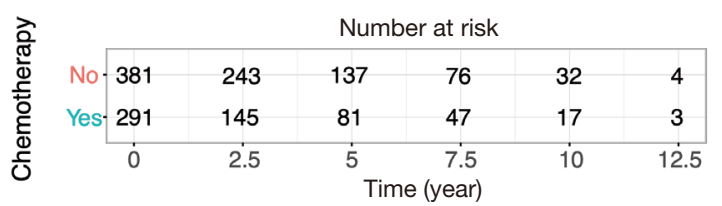

Figure 3 Overall and cancer-specific survival rates of ANMA patients receiving or otherwise on post-operative chemotherapy (A) overall and (B) cancer-specific. ANMA, appendiceal non-mucinous adenocarcinomas.

the impact of pathologic grade was insignificant. We further assessed the prognostic value of post-operative chemotherapy between these four groups of patients (metastatic verses non metastatic and well differentiated verses poorly differentiated). We found post-operative chemotherapy only offers significant benefits to patients with both metastatic and well differentiated ANMA. The 3 -year OS for this group of patients increased from $47.8 \%$ to $57.5 \%$, whereas the CSS increased from $49.8 \%$ to $58.1 \%$. Meanwhile, the 5 -year OS increased from $38.7 \%$ to $47.8 \%$, whereas CSS increased from $39.3 \%$ to $48.1 \%$ (Figure 6). Notably, regardless of metastatic status, chemotherapy offers no significant benefit in poorly differentiated tumors. It's worth mentioned that patients with Stage III ANMA had lymphatic metastases and patients with Stage IV ANMA had widespread metastases, so they may receive different post-operative chemotherapy projects. Thus, we further explored the benefit on survival for both of them in the context of well differentiate histology. We found postoperative chemotherapy only offers significant benefits to patients with well differentiated Stage IV ANMA (Figure 7). Especially, the 3-year OS for this group of patients increased from $29.2 \%$ to $41.1 \%$, whereas the CSS increased from $29.2 \%$ to $40.7 \%$. Meanwhile, the 5 -year OS increased from $21.9 \%$ to $29.7 \%$, whereas CSS increased from $21.9 \%$ to $28.7 \%$. The effect of post-operative chemotherapy on the survival rate in each group of patients is summarized in Table 3.

\section{Discussion}

In general, ANMA patients suffer significantly poor prognosis than their AMA counterparts. Multiple studies have reported on advances in AMA treatment (24-26). Currently, CRS and hyperthermic intraperitoneal chemotherapy (HIPEC) have been shown to offer best treatment for AMA (27-29). However, the management of ANMA patients remains a challenge. For instance, even though surgery is the undisputed preferred treatment, the preferential use of post-operative chemotherapy regimen in ANMA patients remains controversial, thanks to limited systematic clinical studies on the cancer. In this research, metastasis and poor pathological differentiation were the most significant factors for poor prognosis, consistent with previous findings (14). Multivariate Cox analysis combining the disease stage and pathology grade showed that postoperative chemotherapy offered no survival advantage to ANMA patients (Table 2).

According to NCCN, chemotherapy for ANMA should be administered in line with colon cancer guidelines, which 
A

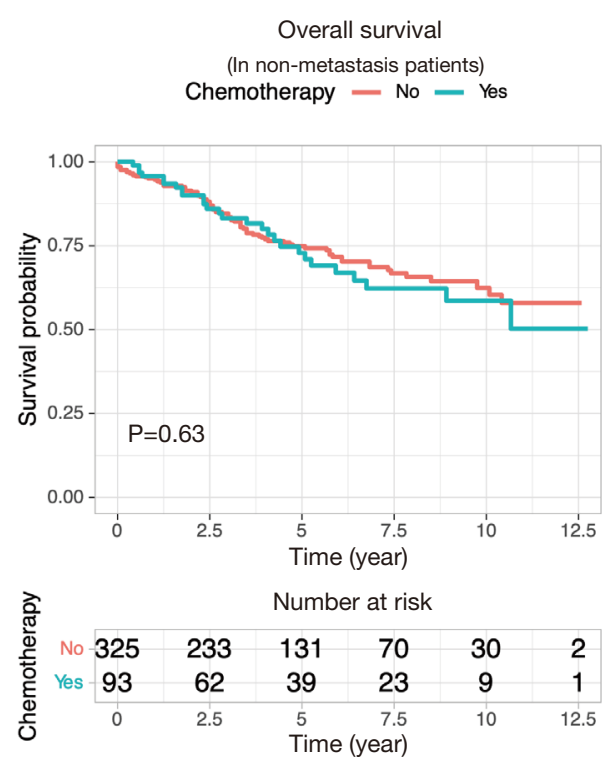

C

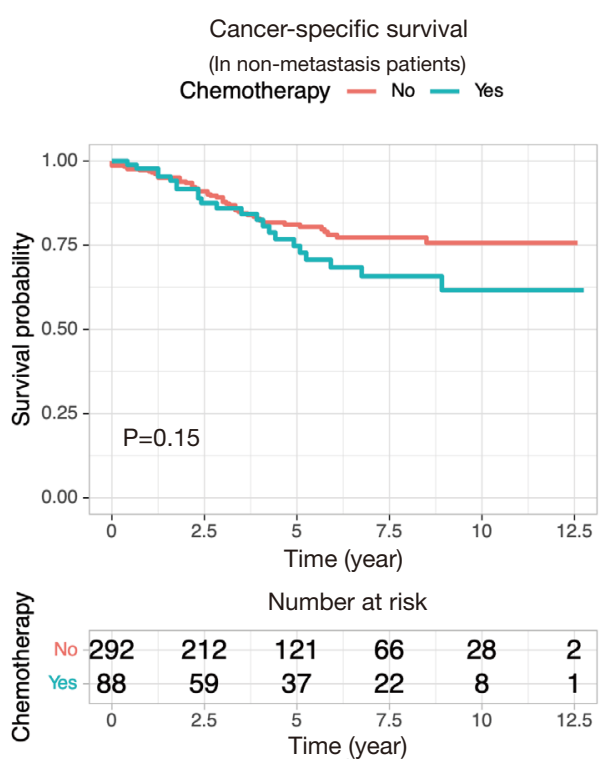

B
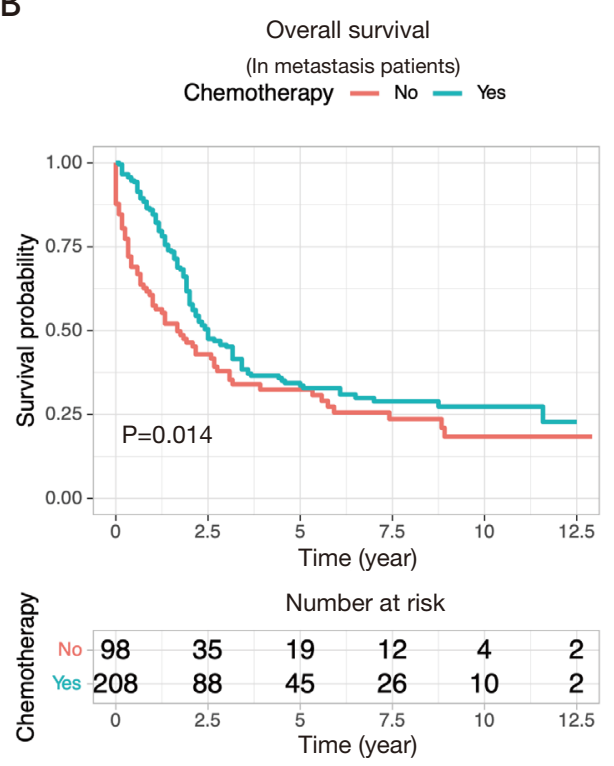

$\mathrm{D}$

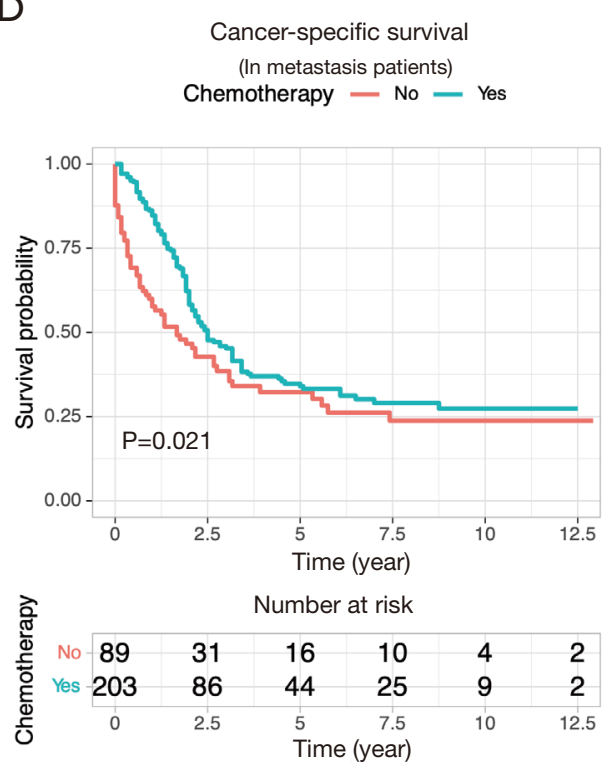

Figure 4 Survival of ANMA patients receiving or not receiving post-operative chemotherapy stratified along disease stage (A,B) overall and (C,D) cancer-specific (A,C non-metastasis; B,D metastasis). ANMA, appendiceal non-mucinous adenocarcinomas.

recommends post-operative chemotherapy for patients with Stage III-IV (metastasis) or with palpable risk factors $(17,30,31)$. According to our analysis, ANMA patients with metastasis were 7.42 times more likely to receive postoperative chemotherapy than those with non-metastatic types. This underlines the sameness in the rationale for chemotherapy in colorectal cancer and ANMA (17). Thus, we further analyzed the survival benefit of chemotherapy based on tumor stage. For the non-metastasis group, postoperative chemotherapy offered no survival advantage, but it seemingly benefited patients with metastatic tumors. Even so, it did not significantly improve the 5 -year survival rate of patients. In addition, patients with poorly differentiated tumors were 2.10 times more likely to receive postoperative chemotherapy, compared to their counterparts. Considering that ANMA is highly heterogenous, 
A

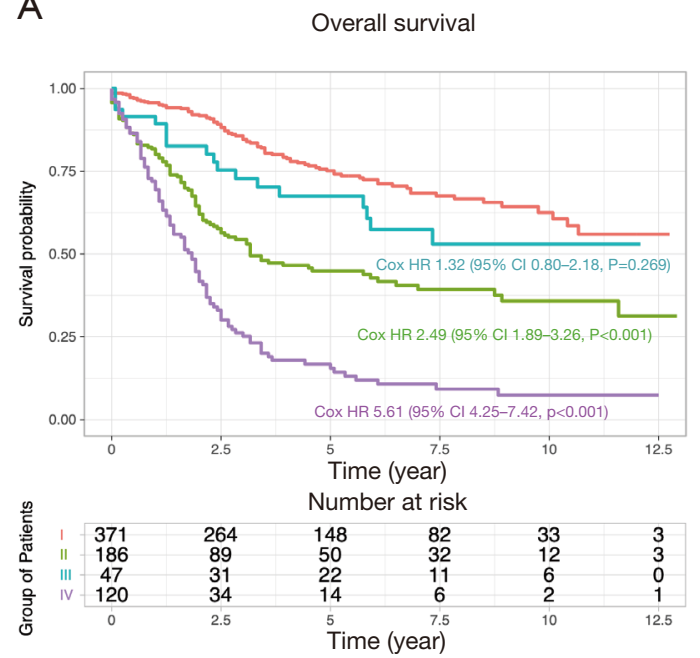

B

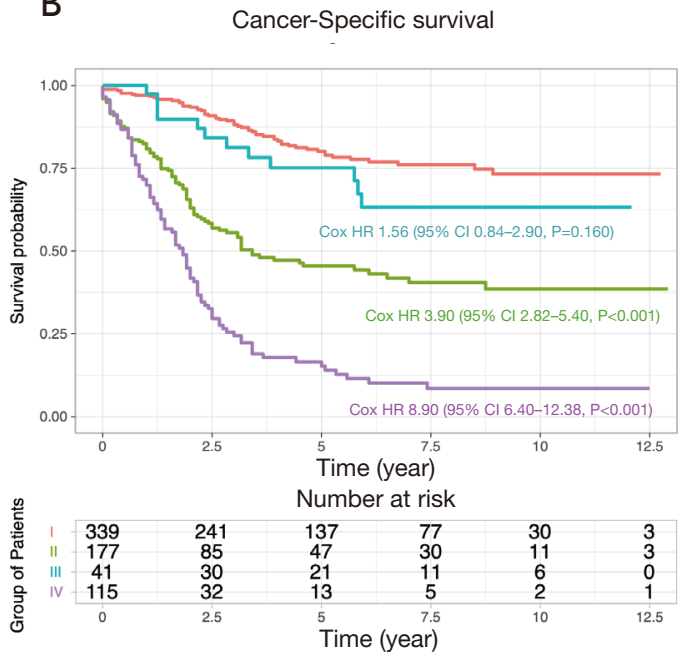

Group of Patients

I: Non-metastasis + Well Differentiation

- II: Metastasis + Well Differentiation

III: Non-metastasis + Poor Differentiation

IV: Metastasis + Poor Differentiation.

Figure 5 Survival of ANMA patients stratified by disease stage and pathological grade (A) overall and (B) cancer-specific. ANMA, appendiceal non-mucinous adenocarcinomas.

histological grade may also guide the administration of postoperative chemotherapy. Therefore, we further analyzed the prognostic benefit of post-operative chemotherapy on well-differentiated $v s$. non-well-differentiated tumors, along the metastatic and non-metastatic stratus. We found postoperative chemotherapy only benefited patients with both metastatic and well-differentiated ANMA tumors.

According to the $6^{\text {th }}$ editions of AJCC staging system for appendix cancer, patients with Stage III had lymphatic metastases and patients with Stage IV had widespread metastases. Typically, patients with Stage III ANMA undergo R0 (curative) surgery and are more likely to receive adjuvant chemotherapy, whereas Stage IV patients usually undergo R2 surgery and are more likely to be given conventional chemotherapy. Therefore, we evaluated the benefits of surgery combined with chemotherapy for specific types of patients with metastatic ANMA, as they may receive different chemotherapy regimens. Our study showed that in post-operative patients with well-differentiated ANMA, the Stage IV patients get both of longer OS and CSS from chemotherapy, the Stage IIIB patients only show an improved OS, and other patients did not benefit from chemotherapy. Thus, the adjuvant chemotherapy may have a limited improvement in patient prognosis, but performing conventional chemotherapy is effective for patients with distant metastatic well-differentiated ANMA.

Although one single-center study suggested that adjuvant chemotherapy was highly beneficial against poordifferentiated appendiceal cancer, the evaluation was only performed on patients who underwent CRS, with no additional evaluation along ANMA subtypes (32). In addition, one previous study revealed that adjuvant chemotherapy had no significant prognostic benefit for AMA patients with metastasis and well-differentiated tumors (13), comparable to our findings. This underlines the inherent differential response of various appendiceal adenocarcinoma subtypes to chemotherapies.

Unfortunately, based on SEER database, effective chemotherapy regimens are still lacking, similar to appendix cancer. Meanwhile, a next-generation sequencing based study revealed that the mutational profile of ANMA was comparable to that of CRC, which share alteration in TP53, KRAS, BRAF and PIK3CA genes (33). Therefore, 5 -fluorouracil-based chemotherapy regimens, used against CRC, have been widely adopted for ANMA, guided by genetic rational between the two cancers (34). In addition, the predictive value of microsatellite instability (MSI) pertaining chemotherapy is significant, particularly in 

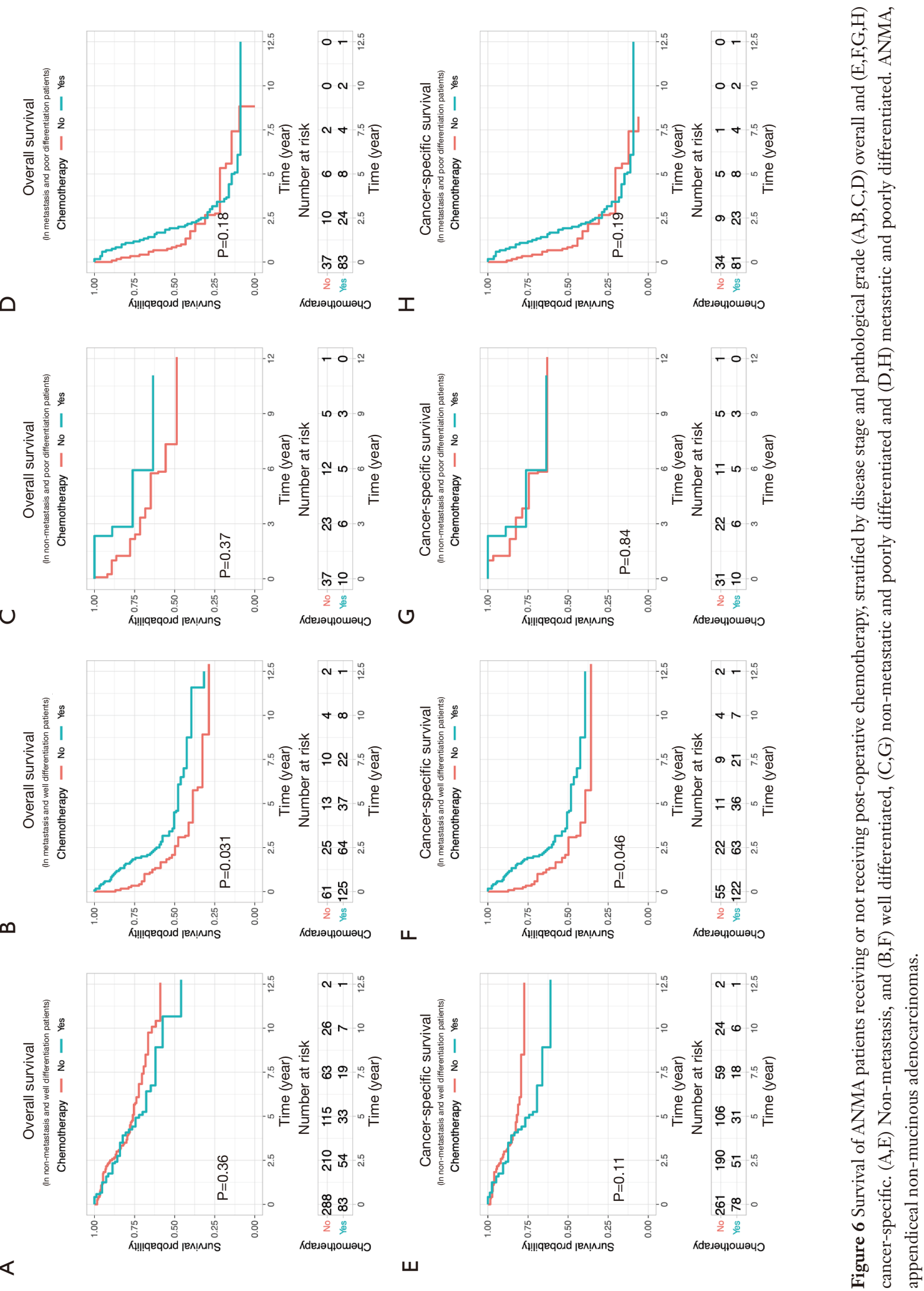


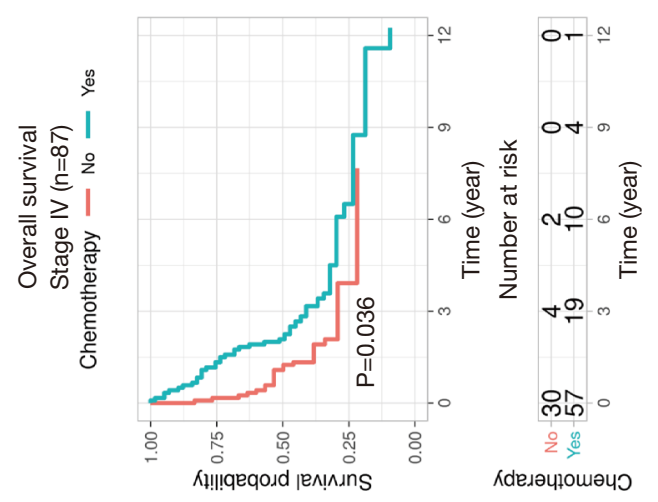

口
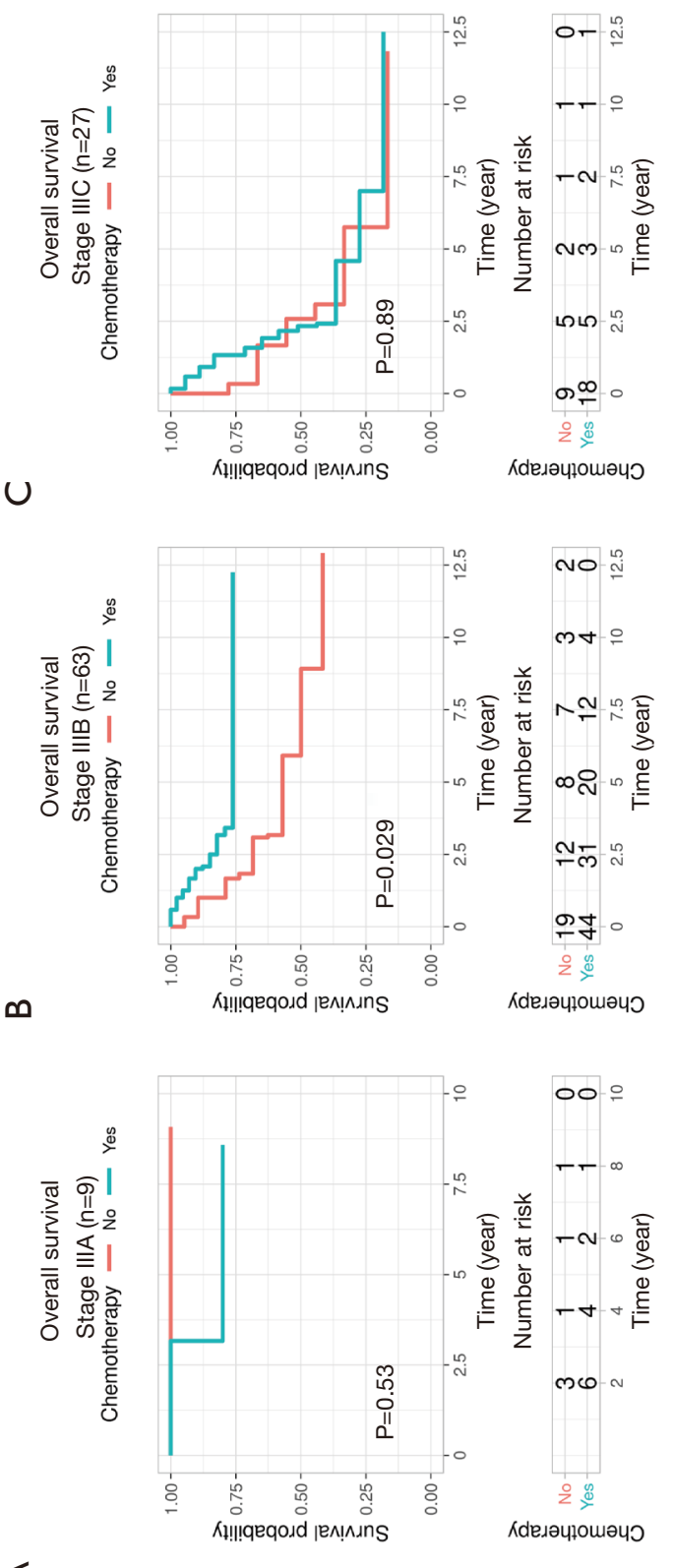

$\varangle$

C A Annals of Palliative Medicine. All rights reserved.

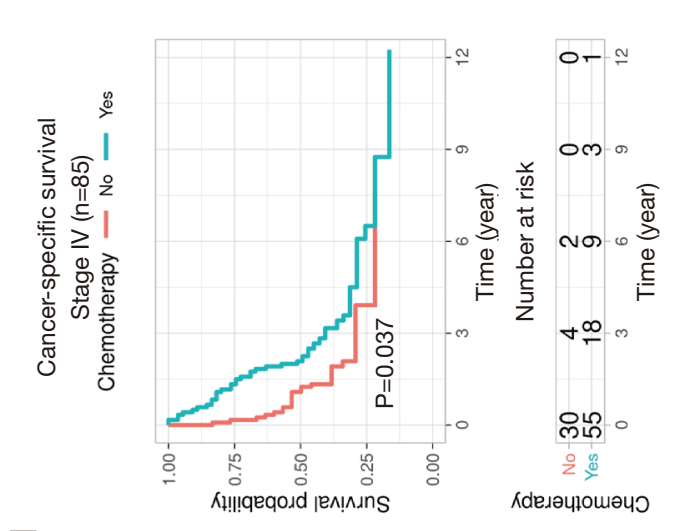

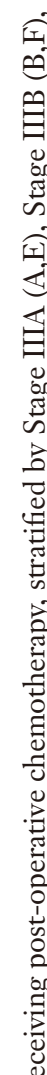

$\circlearrowleft$
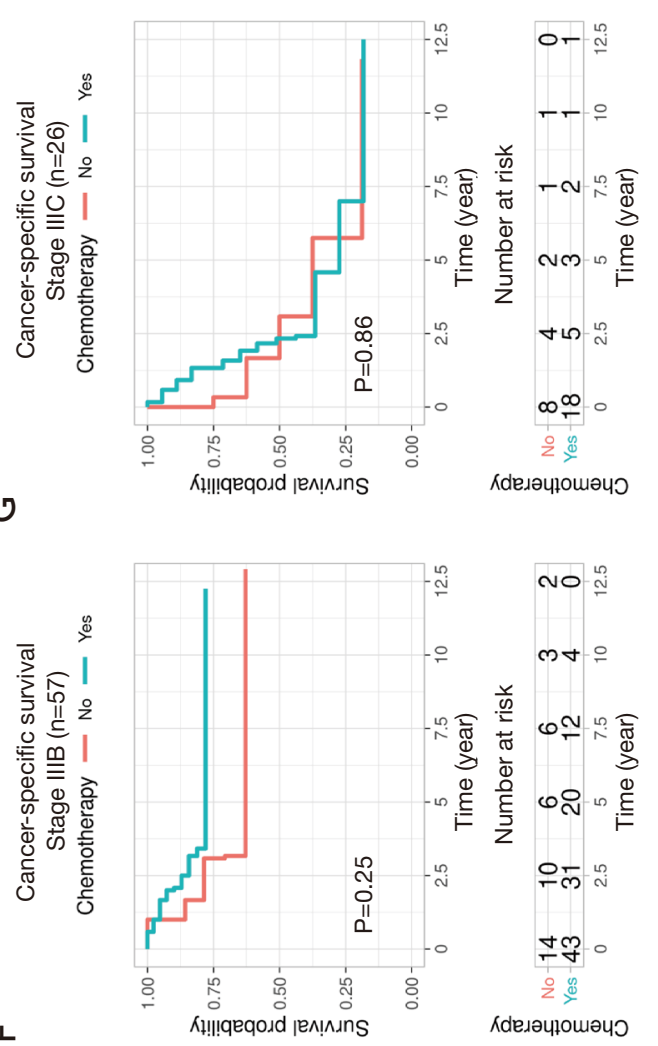

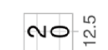
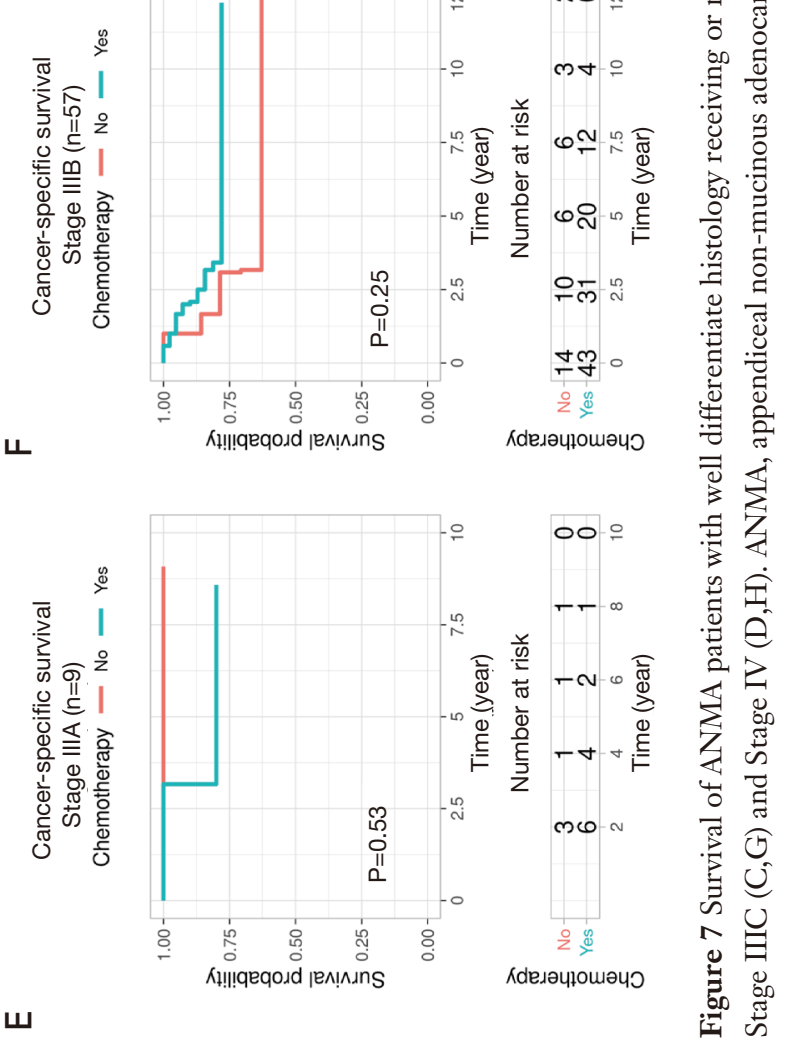

Ann Palliat Med 2021;10(5):5156-5170 | http://dx.doi.org/10.21037/apm-20-2233 
Table 3 The survival period of ANMA patients stratified along disease stage and pathological grade

\begin{tabular}{|c|c|c|c|c|c|c|c|c|}
\hline Patient stratification & \multicolumn{4}{|c|}{ OS } & \multicolumn{4}{|c|}{ css } \\
\hline \multicolumn{9}{|l|}{ Disease stage } \\
\hline I-II (non-metastasis) & Yes & 93 & 83.1 & 72.8 & Yes & 88 & 87.8 & 81.1 \\
\hline III-IV (metastasis) & No & 98 & 37.9 & 32.5 & No & 89 & 38.5 & 32.2 \\
\hline \multicolumn{9}{|l|}{ Disease stage and pathologic grade } \\
\hline $\begin{array}{l}\text { Non-metastasis + well } \\
\text { differentiation }\end{array}$ & Yes & 83 & 84.1 & 72.1 & Yes & 78 & 87.3 & 74.3 \\
\hline \multirow{2}{*}{$\begin{array}{l}\text { Non-metastasis + poor } \\
\text { differentiation }\end{array}$} & Yes & 125 & 57.5 & 47.8 & Yes & 122 & 58.1 & 48.1 \\
\hline & No & 61 & 47.8 & 38.7 & No & 55 & 49.8 & 39.3 \\
\hline \multirow[t]{2}{*}{ Metastasis + poor differentiation } & Yes & 83 & 26.5 & 12.6 & Yes & 81 & 25.8 & 13 \\
\hline & No & 37 & 21.7 & 21.7 & No & 34 & 20.5 & 20.5 \\
\hline \multicolumn{9}{|l|}{ Metastasis in well differentiate ANMA } \\
\hline \multirow[t]{2}{*}{ Stage III } & Yes & 68 & 72.2 & 63.9 & Yes & 67 & 73.3 & 65.0 \\
\hline & No & 31 & 64.4 & 53.6 & No & 25 & 72.0 & 58.5 \\
\hline
\end{tabular}

ANMA, appendiceal non-mucinous adenocarcinomas; OS, overall survival; CSS, cancer-specific survival.

5-fluorouracil resistant cancers (35). Therefore, CRC MSI was divided into MSI high (MSI-H) and low (MSI-L), where patients with MSI-H CRC were found to be more responsive to chemotherapy, thus exhibit better prognosis. MSI was also significant in appendiceal malignant tumors, albeit at 2.8\% (36). Meanwhile, Angélica et al. reported that after surgery, patients with appendix cancer exhibiting MSI benefited from 10 cycles of FOLFOX post-operative chemotherapy. This demonstrates that MSI pattern in appendix cancer may guide the choice of chemotherapy (37). A separate study found that VEGF expression is associated with poor prognosis of appendix cancer, supporting the role of anti-VEGF therapy (38). Recently, one retrospective study showed that bevacizumab, an anti-VEGF agent approved in combination with chemotherapy in CRC, when combined systemic chemotherapy, can benefit patients with appendix cancer but who cannot undergo surgery (39).
Overall, our study provides an intriguing revelation, upon which further clinical trials should built, with the aim of improving ANMA treatment. Because ANMA is a rare cancer, collaborative research may provide large informative data within a shorter period.

Being a retrospective study, our research suffered several limitations. First, our study may be subject to selection bias, because the majority of the patients in this study were drawn from one population. Second, the response to chemotherapy may be influenced by other confounding factors of underlying medical conditions such as hypertension or diabetes that may influence the choice of post-operative chemotherapy. Third, due to the rarity of appendix cancer, we only analyzed less than 1,000 cases. As such, the small sample size may overestimate the statistical findings. In addition, the SEER database lacked information on duration, sequence and specific chemotherapy regimen. 
Limitations notwithstanding, ANMA is a rare disease, which constrains collection of adequate data for strong statistical analyses. Even so, our study provides preliminary findings to guide the rationale in the application of postoperative chemotherapy in ANMA patients.

\section{Conclusions}

Taken together, disease stage and pathological grade impacts on the management of ANMA, particularly on the choice of post-operative chemotherapy. Based on the findings of this research, we report that only patients with well differentiated and widespread metastatic ANMA can benefit from post-operative chemotherapy. Nonetheless, more prospective multidisciplinary and collaborative clinical trials are necessary to fully understand the pathological signature of ANMA that may effectively respond to this therapy.

\section{Acknowledgments}

The authors are grateful for the language editing of native English-speaking expert Julio Erickson from freescience Inc. We also appreciate the invaluable support and useful discussions with other members of the general surgery department.

Funding: This research was funded by the Health Planning Project of Shanghai Pudong New Area Health and Family Planning Committee (Grant No. PW2020A-58) and the Talent Project of Shanghai Pudong New Area Gongli Hospital (Grant No. GLRq2020-07).

\section{Footnote}

Reporting Checklist: The authors have completed the STROBE reporting checklist. Available at http://dx.doi. org/10.21037/apm-20-2233

Conflicts of Interest: All authors have completed the ICMJE uniform disclosure form (available at http://dx.doi. org/10.21037/apm-20-2233). The authors have no conflicts of interest to declare.

Ethical Statement: The authors are accountable for all aspects of the work in ensuring that questions related to the accuracy or integrity of any part of the work are appropriately investigated and resolved. The study was conducted in accordance with the Declaration of Helsinki (as revised in 2013).
Open Access Statement: This is an Open Access article distributed in accordance with the Creative Commons Attribution-NonCommercial-NoDerivs 4.0 International License (CC BY-NC-ND 4.0), which permits the noncommercial replication and distribution of the article with the strict proviso that no changes or edits are made and the original work is properly cited (including links to both the formal publication through the relevant DOI and the license). See: https://creativecommons.org/licenses/by-nc-nd/4.0/.

\section{References}

1. Marmor S, Portschy PR, Tuttle TM, et al. The Rise in Appendiceal Cancer Incidence: 2000-2009. J Gastrointest Surg 2015;19:743-50.

2. Overman MJ, Fournier $\mathrm{K}, \mathrm{Hu} \mathrm{CY}$, et al. Improving the AJCC/TNM staging for adenocarcinomas of the appendix: the prognostic impact of histological grade. Ann Surg 2013;257:1072-8.

3. Whitfield CG, Amin SN, Garner JP. Surgical management of primary appendiceal malignancy: Surgical management of primary appendiceal malignancy. Colorectal Dis 2012;14:1507-11.

4. McGory ML, Maggard MA, Kang H, et al. Malignancies of the Appendix: Beyond Case Series Reports. Dis Colon Rectum 2005;48:2264-71.

5. Carr NJ, Finch J, Ilesley IC, et al. Pathology and prognosis in pseudomyxoma peritonei: a review of 274 cases. J Clin Pathol 2012;65:919.

6. Pai RK, Longacre TA. Appendiceal Mucinous Tumors and Pseudomyxoma Peritonei: Histologic Features, Diagnostic Problems, and Proposed Classification. Adv Anat Pathol 2005;12:291-311.

7. Panarelli NC, Yantiss RK. Mucinous Neoplasms of the Appendix and Peritoneum. Arch Pathol Lab Med 2011;135:1261-8.

8. Tsikitis VL. "Miscellaneous" Tumors of the Small Bowel and Colon and Rectum. Clin Colon Rectal Surg 2018;31:265-66.

9. Guzman C, Boddhula S, Panneerselvam N, et al. Appendiceal Carcinoid Tumors: Is There a Survival Advantage to Colectomy over Appendectomy? J Gastrointest Surg 2020;24:1149-57.

10. Turaga K, Levine E, Barone R, et al. Consensus Guidelines from The American Society of Peritoneal Surface Malignancies on Standardizing the Delivery of Hyperthermic Intraperitoneal Chemotherapy (HIPEC) in Colorectal Cancer Patients in the United States. Ann Surg Oncol 2014;21:1501-5. 
11. Lu P, Fields AC, Meyerhardt JA, et al. Systemic chemotherapy and survival in patients with metastatic low-grade appendiceal mucinous adenocarcinoma. J Surg Oncol 2019;120:446-51.

12. Lieu CH, Lambert LA, Wolff RA, et al. Systemic chemotherapy and surgical cytoreduction for poorly differentiated and signet ring cell adenocarcinomas of the appendix. Ann Oncol 2012;23:652-8.

13. Asare EA, Compton CC, Hanna NN, et al. The impact of stage, grade, and mucinous histology on the efficacy of systemic chemotherapy in adenocarcinomas of the appendix: Analysis of the National Cancer Data Base. Cancer 2016;122:213-21.

14. Uemura M, Qiao W, Fournier K, et al. Retrospective study of nonmucinous appendiceal adenocarcinomas: role of systemic chemotherapy and cytoreductive surgery. BMC Cancer 2017;17:331.

15. Sugarbaker PH, Chang D. Results of Treatment of 385 Patients With Peritoneal Surface Spread of Appendiceal Malignancy. Ann Surg Oncol 1999;6:727-31.

16. Ma R, Wang B, Zhai X, et al. Management and prognostic prediction of appendiceal mucinous adenocarcinoma with peritoneal metastasis: a single center study in China. BMC Cancer 2020;20:280.

17. Tejani MA, Veer A, Milne D, et al. Systemic therapy for advanced appendiceal adenocarcinoma: an analysis from the NCCN Oncology Outcomes Database for colorectal cancer. J Natl Compr Canc Netw 2014;12:1123-30.

18. Ang CS, Shen JP, Hardy-Abeloos CJ, et al. Genomic Landscape of Appendiceal Neoplasms. JCO Precis Oncol 2018;2:PO.17.00302.

19. Shen JP, Jacobs MT, Fuh IL, et al. Somatic mutation landscape of appendiceal cancer. J Clin Oncol 2018;36:671.

20. Lou X, Li J, Wei Y-Q, et al. Comparable prevalence of distant metastasis and survival of different primary site for LN + pancreatic tumor. J Transl Med 2020;18:266.

21. Mao W, Wei S, Yang H, et al. Clinicopathological study of organ metastasis in endometrial cancer. Future Oncol 2020;16:525-40.

22. He C, Cai Z, Zhang Y, et al. Prognostic Model to Predict Cancer-Specific Survival for Patients With Gallbladder Carcinoma After Surgery: A Population-Based Analysis. Front Oncol 2019;9:1329.

23. Jiang S, Zhen H, Jiang H. Second primary malignancy in diffuse large B-cell lymphoma patients: A SEER database analysis. Curr Probl Cancer 2020;44:100502.

24. Winder T, Lenz H. Mucinous Adenocarcinomas with Intra-Abdominal Dissemination: A Review of Current
Therapy. Oncologist 2010;15:836-44.

25. Carr NJ, Bibeau F, Bradley RF, et al. The histopathological classification, diagnosis and differential diagnosis of mucinous appendiceal neoplasms, appendiceal adenocarcinomas and pseudomyxoma peritonei. Histopathology 2017;71:847-58.

26. Dulskas A, Poskus T, Poskus E, et al. Long-Term Outcomes after Surgery for Appendiceal Mucinous Tumours. Visc Med 2018;34:151-5.

27. Di Leo A, Corvasce A, Weindelmayer J, et al. Cytoreductive surgery (CRS) and hyperthermic intraperitoneal chemotherapy (HIPEC) in pseudomyxoma peritonei of appendiceal origin: result of a single centre study. Updates Surg 2020;72:1207-12.

28. Andrea P, Duy N, Jeffrey F, et al. The Role of Heated Intraperitoneal Chemotherapy (HIPEC) in Low-Grade Appendiceal Neoplasm: Friend or Foe? J Gastrointest Dig Syst 2016;6:1-4.

29. Solomon D, Bekhor E, Leigh N, et al. Surveillance of LowGrade Appendiceal Mucinous Neoplasms With Peritoneal Metastases After Cytoreductive Surgery and Hyperthermic Intraperitoneal Chemotherapy: Are 5 Years Enough? A Multisite Experience. Ann Surg Oncol 2020;27:147-53.

30. Messersmith WA. Systemic Management of Colorectal Cancer. J Natl Compr Canc Netw 2017;15:699-702.

31. Benson AB 3rd, Venook AP, Cederquist L, et al. Colon Cancer, Version 1.2017, NCCN Clinical Practice Guidelines in Oncology. J Natl Compr Canc Netw 2017;15:370-98.

32. Kolla BC, Petersen A, Chengappa M, et al. Impact of adjuvant chemotherapy on outcomes in appendiceal cancer. Cancer Med 2020;9:3400-6.

33. Jesinghaus M, Konukiewitz B, Foersch S, et al. Appendiceal goblet cell carcinoids and adenocarcinomas ex-goblet cell carcinoid are genetically distinct from primary colorectal-type adenocarcinoma of the appendix. Mod Pathol 2018;31:829-39.

34. Shapiro JF, Chase JL, Wolff RA, et al. Modern systemic chemotherapy in surgically unresectable neoplasms of appendiceal origin. Cancer 2010;116:316-22.

35. Arnold CN, Goel A, Boland CR. Role of hMLH1 promoter hypermethylation in drug resistance to 5fluorouracil in colorectal cancer cell lines. Int J Cancer 2003;106:66-73.

36. Taggart MW, Galbincea J, Mansfield PF, et al. High-level Microsatellite Instability in Appendiceal Carcinomas. Am J Surg Pathol 2013;37:1192-200.

37. Morales-Miranda A, Rosado ID, Núñez CC, et al. 
Appendiceal carcinoma associated with microsatellite instability. Mol Clin Oncol 2018;8:694-8.

38. Logan-Collins JM, Lowy AM, Robinson-Smith TM, et al. VEGF Expression Predicts Survival in Patients with Peritoneal Surface Metastases from Mucinous Adenocarcinoma of the Appendix and Colon. Ann Surg
Oncol 2008;15:738-44.

39. Choe JH, Overman MJ, Fournier KF, et al. Improved Survival with Anti-VEGF Therapy in the Treatment of Unresectable Appendiceal Epithelial Neoplasms. Ann Surg Oncol 2015;22:2578-84.

Cite this article as: Yu T, Fan H, Lv Q, Li J, Wei L, Wang M, Dong J, Cai X. Post-operative chemotherapy improves the survival of patients with well differentiated widespread metastatic appendiceal non-mucinous adenocarcinomas. Ann Palliat Med 2021;10(5):5156-5170. doi: 10.21037/apm-20-2233 